PROCEEDINGS OF THE

AMERICAN MATHEMATICAL SOCIETY

Volume 135, Number 6, June 2007, Pages 1725-1734

S 0002-9939(06)08647-3

Article electronically published on November 14, 2006

\title{
MIDDLE POINTS, MEDIANS AND INNER PRODUCTS
}

\author{
CARLOS BENÍTEZ AND DIEGO YÁÑEZ
}

(Communicated by N. Tomczak-Jaegermann)

\begin{abstract}
Let $X$ be a real normed space with unit sphere $S$. Gurari and Sozonov proved that $X$ is an inner product space if and only if, for any $u, v \in S$, $\inf _{t \in[0,1]}\|t u+(1-t) v\|=\left\|\frac{1}{2} u+\frac{1}{2} v\right\|$. We prove that it suffices to consider points $u, v \in S$ such that $\inf _{t \in[0,1]}\|t u+(1-t) v\|=\frac{1}{2}$.

Making use of the above result we also prove that if $\operatorname{dim} X \geq 3, X$ is smooth, and 0 is a Fermat-Torricelli median of any three points $u, v, w \in S$ such that $u+v+w=0$, then $X$ is an inner product space.
\end{abstract}

\section{INTRODUCTION}

Let $X$ be a real normed space with unit sphere $S$. Gurari and Sozonov [8] proved that $X$ is an inner product space (i.p.s.) if and only if, for any $u, v \in S$,

$$
\inf _{t \in[0,1]}\|t u+(1-t) v\|=\left\|\frac{1}{2} u+\frac{1}{2} v\right\|
$$

(see, e.g., 11, p. 29, where this result is used to establish many characterizations of i.p.s., especially in chapters 12 to 19 ). We prove in this paper that it suffices to consider pairs of points $u, v \in S$ such that

$$
\inf _{t \in[0,1]}\|t u+(1-t) v\|=\frac{1}{2}
$$

i.e., we prove that $X$ is an i.p.s. if and only if

$$
u, v \in S, \inf _{t \in[0,1]}\|t u+(1-t) v\|=\frac{1}{2} \Rightarrow u+v \in S .
$$

In geometrical terms, property (1) states that every chord of $S$ that supports $\frac{1}{2} S$ touches $\frac{1}{2} S$ at its middle point.

As a corollary of the above result, we obtain a new characterization of i.p.s. based on the location of the medians of three points.

By definition, the set $Z_{L}(u, v, w)$ of the Fermat-Torricelli medians of the points $u, v, w \in X$ from the set $L \subset X$ is formed by the points $z \in L$ such that

$$
\|u-z\|+\|v-z\|+\|w-z\|=\inf _{x \in L}(\|u-x\|+\|v-x\|+\|w-x\|) .
$$

It is well known (see, e.g., 4, p. 274, or [6, p. 98) that if $X$ is either an i.p.s. or a two-dimensional space, then, for every $u, v, w \in X$,

$$
Z_{X}(u, v, w)=Z_{\mathrm{aff}(u, v, w)}(u, v, w)=Z_{\mathrm{co}(u, v, w)}(u, v, w),
$$

Received by the editors July 13, 2005 and, in revised form, December 26, 2005.

2000 Mathematics Subject Classification. Primary 49B20, 46C15, $90 \mathrm{~B} 85$.

This work was partially supported by MEC (Spain) and FEDER (UE), MTM2004-06226.

(C)2006 American Mathematical Society 
where $\operatorname{aff}(u, v, w)$ and $\operatorname{co}(u, v, w)$ are the affine and the convex hull, respectively, of the points $u, v, w \in X$.

However, the formally weaker property

$$
Z_{X}(u, v, w) \cap \operatorname{co}(u, v, w) \neq \emptyset, \quad \text { for every } u, v, w \in X,
$$

is characteristic of real i.p.s. of dimension $\geq 3$ 2.

Also it is known (see, e.g., 4, p. 238, or the proof of Theorem 3.2 of this paper) that if $X$ is an i.p.s., then

$$
u, v, w \in S, u+v+w=0 \Rightarrow 0 \in Z_{X}(u, v, w) .
$$

We prove in the above-mentioned Theorem 3.2 that property (2), when $X$ is smooth and of dimension $\geq 3$, is also characteristic of i.p.s..

\section{Preliminary Lemmas}

It follows from the nature of property (1) and the fact that $X$ is an i.p.s. when its two-dimensional subspaces are, that it suffices to consider the case in which $X$ is two-dimensional, i.e., the space $\mathbb{R}^{2}$ endowed with a norm with unit sphere $S$ and unit ball $B$.

For given $x=\left(x_{1}, x_{2}\right)$ and $y=\left(y_{1}, y_{2}\right)$ in $X$ we shall use the following notation:

$x \prec y$, when $x$ precedes $y$ in the positive orientation of $X$, i.e.,

$$
x \wedge y=x_{1} y_{2}-x_{2} y_{1}>0 .
$$

$x \perp y$, when $x$ is orthogonal to $y$ in the sense of Birkhoff, 3], 9, i.e.,

$$
\|x\| \leq\|x+\lambda y\| \quad(\lambda \in \mathbb{R}),
$$

or, equivalently (see, e.g., [7]),

$$
|x \wedge y|=\sup \{z \wedge y:\|z\|=\|x\|\} .
$$

Obviously, $x \perp y$ means that the straight line $L=\{x+\lambda y: \lambda \in \mathbb{R}\}$ supports the sphere $S(0,\|x\|)$ at $x$, i.e., $x \in L \cap S(0,\|x\|)$ and $L \cap \operatorname{int} B(0,\|x\|)=\emptyset$.

Lemma 2.1. (i) For any $u \in S$, there are unique $u^{*}, u^{* *} \in S, u^{* *} \prec u \prec u^{*}$, such that the segments $\left[u^{* *}, u\right]$ and $\left[u, u^{*}\right]$ support $\frac{1}{2} S$.

(ii) The map $u \in S \rightarrow u^{*} \in S$ is a homeomorphism whose inverse is $u \in S \rightarrow$ $u^{* *} \in S$.

(iii) If $u, v \in S$ are such that $u \prec v$, then, $u^{*} \prec v^{*}$ and $u+u^{*} \prec v+v^{*}$.

Proof. The proof is very intuitive and not difficult.

Remark 2.2. $\left[u, u^{*}\right]$ supports $\frac{1}{2} S$ means that $\left[u, u^{*}\right] \cap \frac{1}{2} S \neq \emptyset$ and, for any $x \in$ $\left[u, u^{*}\right] \cap \frac{1}{2} S, x \perp u^{*}-u$. In other words, $x^{\prime}\left(u^{*}-u\right)=0$ when $x^{\prime}$ is some linear functional which attains its norm at $x$.

Lemma 2.3. If $X$ fulfills (1), then it is regular (rotund and smooth).

Proof. Suppose that $X$ is non-rotund. Then there exist $u \in S$ and $x, y \in \frac{1}{2} S, x \prec y$, such that $\left[u, u^{*}\right] \cap \frac{1}{2} S=[x, y]$.

Property (1) states that $\left[u, u^{*}\right]$ supports $\frac{1}{2} S$ at $\frac{1}{2}\left(u+u^{*}\right)$ and, hence, $x \preceq u+u^{*} \preceq$ $y$. Suppose that $u+u^{*} \prec y$. It follows from Lemma 2.1 that there exists $v \in S$ such that $u \prec v$ and $u+u^{*} \prec v+v^{*} \prec y$. Then the fact that $\left[v, v^{*}\right]$ supports $\frac{1}{2} S$ at $\frac{1}{2}\left(v+v^{*}\right)$ leads to the absurdity $\left[v, v^{*}\right] \cap \frac{1}{2} S=\left[u, u^{*}\right] \cap \frac{1}{2} S=[x, y]$. 
Suppose now that $X$ is non-smooth. Then there are $u, v \in S, u \prec v$, such that $\frac{1}{2}\left(u+u^{*}\right)=\frac{1}{2}\left(v+v^{*}\right)$ is the only point of $\left[u, u^{*}\right] \cap \frac{1}{2} S=\left[v, v^{*}\right] \cap \frac{1}{2} S$. Hence, the segments $[u, v]$ and $\left[u^{*}, v^{*}\right]$ must be contained in $S$, in contradiction with the first part of this lemma.

Corollary 2.4. If $X$ fulfills (1) and $u, v \in S$ are such that $u \prec v$ and $u+v \in S$, then $v=u^{*}$.

Proof. Let (other cases will be analogous) $u^{*} \prec v$ and $u \wedge u^{*} \leq u \wedge v$. Since $\left(u+u^{*}\right) \wedge u^{*}>0, u^{*} \wedge v>0$, and $\left(u+u^{*}\right) \wedge v>0$ (i.e., $u^{*}$ is between $u+u^{*}$ and $v)$, there exist $0<t<1$ and $\rho>0$ such that $\rho u^{*}=t\left(u+u^{*}\right)+(1-t) v$. Then, $\rho u \wedge u^{*}=t u \wedge u^{*}+(1-t) u \wedge v \geq u \wedge u^{*}$ and hence $\rho \geq 1$.

Therefore, it follows from $u+u^{*}, v \in S$ that $\rho=1$ and the segment $\left[u+u^{*}, v\right]$ is in $S$, in contradiction with the rotundity of $X$.

Lemma 2.5. Suppose that $X$ fulfills (1). Then, for every $u \in S$,

(i) $\left(u^{*}\right)^{*}=u^{* *}$.

(ii) $u+u^{*}+u^{* *}=0$.

(iii) $u \wedge u^{*}=u^{*} \wedge u^{* *}=u^{* *} \wedge u$.

(iv) $u+u^{*} \perp u-u^{*}, u^{*}+u^{* *} \perp u^{*}-u^{* *}, u^{* *}+u \perp u^{* *}-u$.

Proof. (i) and (ii). It follows from (1) that $-\left(u+u^{*}\right) \in S$, and it is obvious that $u-\left(u+u^{*}\right), u^{*}-\left(u+u^{*}\right) \in S$. Then these properties are an immediate consequence of the above corollary.

(iii). The proof follows from $u \wedge\left(u+u^{*}+u^{* *}\right)=u^{*} \wedge\left(u+u^{*}+u^{* *}\right)=0$.

(iv). It suffices to consider Remark 2.2 and the fact that the segment $\left[u, u^{*}\right]$ supports $\frac{1}{2} S$ at $-\frac{1}{2}\left(u+u^{*}\right)$.

Lemma 2.6. Suppose that $X$ fulfills (1). Then:

(i) For any $u \in S$ there exist unique ${ }^{\perp} u, u^{\perp} \in S,{ }^{\perp} u \prec u \prec u^{\perp}$, such that ${ }^{\perp} u \perp u$ and $u \perp u^{\perp}$. $S$.

(ii) The map $u \in S \rightarrow u^{\perp} \in S$ is a homeomorphism with inverse $u \in S \rightarrow^{\perp} u \in$

(iii) If $u, v \in S$ are such that $u \prec v$, then $u^{\perp} \prec v^{\perp}$.

Proof. (i). It is easy to see and well known [9] that the uniqueness (for every $u \in S$ ) of ${ }^{\perp} u$ and $u^{\perp}$ are equivalent to the rotundity and smoothness of $X$, respectively.

(ii) and (iii). As in Lemma 2.1, the proof is very intuitive and not difficult.

In all that follows

$$
s: \theta \in[0,2 \pi] \rightarrow s(\theta) \in S
$$

will be a "natural map" for $S$, i.e., a map such that $s(\theta)=\left(s_{1}(\theta), s_{2}(\theta)\right)$ is the point of $S$ that makes an angle $\theta$ with a given point $\left(s_{1}(0), s_{2}(0)\right)$ of $S$, measured with the positive orientation of the plane $X$. In other words, if $s(0)=\|(1,0)\|^{-1}(1,0)$, then

$$
s(\theta)=\|(\cos \theta, \sin \theta)\|^{-1}(\cos \theta, \sin \theta) .
$$

Since $S$ is a convex curve, the above map is continuous and of bounded variation, and, as a consequence of Lemma 2.1, also continuous and of bounded variation are the maps (non-natural, in general)

$$
s^{*}: \theta \in[0,2 \pi] \rightarrow s^{*}(\theta) \in S, \quad s^{* *}: \theta \in[0,2 \pi] \rightarrow s^{* *}(\theta) \in S,
$$


where $s^{*}(\theta)$ and $s^{* *}(\theta)$ are the unique points of $S$ such that $s^{* *}(\theta) \prec s(\theta) \prec s^{*}(\theta)$ and the segments $\left[s^{* *}(\theta), s(\theta)\right],\left[s(\theta), s^{*}(\theta)\right]$ support $\frac{1}{2} S$.

Moreover, by virtue of Lemma 2.6. the same holds for

$$
s^{\perp}: \theta \in[0,2 \pi] \rightarrow s^{\perp}(\theta) \in S, \quad{ }^{\perp} s: \theta \in[0,2 \pi] \rightarrow^{\perp} s(\theta) \in S,
$$

when $X$ fulfills (1).

Therefore all the Riemann-Stieltjes integrals that we shall write below make sense. For example, the well-known formula

$$
A\left(B_{s(\alpha) s(\beta)}\right)=\frac{1}{2} \int_{\alpha}^{\beta} s(\theta) \wedge d s(\theta)=\frac{1}{2} \int_{\alpha}^{\beta}\left[s_{1}(\theta) d s_{2}(\theta)-s_{2}(\theta) d s_{1}(\theta)\right]
$$

correctly gives the area of the sector of the unit ball $B$ that is between two points $s(\alpha)$ and $s(\beta)$, with $0 \leq \alpha<\beta \leq 2 \pi$.

Lemma 2.7. If $X$ fulfills (1), then:

(i) For any $u \in S, A\left(B_{u u^{*}}\right)=A\left(B_{u^{*} u^{* *}}\right)=A\left(B_{u^{* *} u}\right)$.

(ii) For any $u, v \in S, A\left(B_{u v}\right)=A\left(B_{u^{*} v^{*}}\right)$.

(iii) The function $u \in S \rightarrow u \wedge u^{*}$ is constant.

Proof. (i). Let

$$
s: \theta \in[0,2 \pi] \rightarrow s(\theta) \in S
$$

be a natural map for $S$ such that $u=s(\alpha)$, and let $0 \leq \alpha<\alpha^{*} \leq 2 \pi$ be such that $s^{*}(\alpha)=s\left(\alpha^{*}\right)$. Then, $s^{* *}(\alpha)=s^{*}\left(\alpha^{*}\right)$ and

$$
\begin{gathered}
2 s(\alpha)=s(\alpha)+s^{*}(\alpha)+\left[s(\alpha)-s^{*}(\alpha)\right], \\
2 s^{*}(\alpha)=s(\alpha)+s^{*}(\alpha)-\left[s(\alpha)-s^{*}(\alpha)\right]=s\left(\alpha^{*}\right)+s^{*}\left(\alpha^{*}\right)+\left[s\left(\alpha^{*}\right)-s^{*}\left(\alpha^{*}\right)\right], \\
2 s^{* *}(\alpha)=s\left(\alpha^{*}\right)+s^{*}\left(\alpha^{*}\right)-\left[s\left(\alpha^{*}\right)-s^{*}\left(\alpha^{*}\right)\right] .
\end{gathered}
$$

Hence,

$$
\begin{gathered}
A\left(B_{u u^{*}}\right)-A\left(B_{u^{*} u^{* *}}\right) \\
=\frac{1}{8} \int_{\alpha}^{\alpha^{*}}\left\{s(\theta)+s^{*}(\theta)+\left[s(\theta)-s^{*}(\theta)\right]\right\} \wedge d\left\{s(\theta)+s^{*}(\theta)+\left[s(\theta)-s^{*}(\theta)\right]\right\} \\
-\frac{1}{8} \int_{\alpha}^{\alpha^{*}}\left\{s(\theta)+s^{*}(\theta)-\left[s(\theta)-s^{*}(\theta)\right]\right\} \wedge d\left\{s(\theta)+s^{*}(\theta)-\left[s(\theta)-s^{*}(\theta)\right]\right\} \\
=\frac{1}{4} \int_{\alpha}^{\alpha^{*}}\left\{\left[s(\theta)+s^{*}(\theta)\right] \wedge d\left[s(\theta)-s^{*}(\theta)\right]+\left[s(\theta)-s^{*}(\theta)\right] \wedge d\left[s(\theta)+s^{*}(\theta)\right]\right\} .
\end{gathered}
$$

Let $\alpha=\theta_{0}<\theta_{1}<\ldots<\theta_{n}=\alpha^{*}$ be a partition of $\left[\alpha, \alpha^{*}\right]$. Since $u+u^{*} \perp u-u^{*}$, (see Lemma 2.5(iv)), there exist $\theta_{0} \leq \delta_{1} \leq \theta_{1} \leq \ldots \leq \theta_{n-1} \leq \delta_{n} \leq \theta_{n}$ such that, for $k=1, \ldots, n$,

$$
\left[s\left(\delta_{k}\right)-s^{*}\left(\delta_{k}\right)\right] \wedge\left\{s\left(\theta_{k}\right)+s^{*}\left(\theta_{k}\right)-\left[s\left(\theta_{k-1}\right)+s^{*}\left(\theta_{k-1}\right)\right]\right\}=0
$$

and, hence,

$$
\int_{\alpha}^{\alpha^{*}}\left[s(\theta)-s^{*}(\theta)\right] \wedge d\left[s(\theta)+s^{*}(\theta)\right]=0
$$


Therefore,

$$
\begin{gathered}
A\left(B_{u u^{*}}\right)-A\left(B_{u^{*} u^{* *}}\right) \\
=\frac{1}{4} \int_{\alpha}^{\alpha^{*}} d\left\{\left[s(\theta)+s^{*}(\theta)\right] \wedge\left[s(\theta)-s^{*}(\theta)\right]\right\}=\frac{1}{2} \int_{\alpha}^{\alpha^{*}} d\left[s^{*}(\theta) \wedge s(\theta)\right] \\
=\frac{1}{2}\left[s(\alpha) \wedge s^{*}(\alpha)-s\left(\alpha^{*}\right) \wedge s^{*}\left(\alpha^{*}\right)\right]=\frac{1}{2}\left[s(\alpha) \wedge s^{*}(\alpha)-s^{*}(\alpha) \wedge s^{* *}(\alpha)\right]=0,
\end{gathered}
$$

where the last equality is justified in Lemma 2.5(iii).

(ii). Suppose (other cases are analogous) that $u, v \in S, u \prec v \prec u^{*}$. Then it suffices to consider (i),

$$
A\left(B_{u u^{*}}\right)=A\left(B_{u^{*} u^{* *}}\right)=A\left(B_{u^{* *} u}\right)=\frac{1}{3} A(B)=A\left(B_{v v^{*}}\right)=A\left(B_{v^{*} v^{* *}}\right)=A\left(B_{v^{* *} v}\right),
$$

and the obvious fact that

$$
A\left(B_{u u^{*}}\right)=A\left(B_{u v}\right)+A\left(B_{v u^{*}}\right), \quad A\left(B_{v v^{*}}\right)=A\left(B_{v u^{*}}\right)+A\left(B_{u^{*} v^{*}}\right) .
$$

(iii). We have proved in (ii) that, for any $0 \leq \alpha<\beta \leq 2 \pi$,

$$
\begin{gathered}
0=A\left(B_{s(\alpha) s(\beta)}\right)-A\left(B_{s^{*}(\alpha) s^{*}(\beta)}\right) \\
=\frac{1}{8} \int_{\alpha}^{\beta}\left\{s(\theta)+s^{*}(\theta)+\left[s(\theta)-s^{*}(\theta)\right]\right\} \wedge d\left\{s(\theta)+s^{*}(\theta)+\left[s(\theta)-s^{*}(\theta)\right]\right\} \\
-\frac{1}{8} \int_{\alpha}^{\beta}\left\{s(\theta)+s^{*}(\theta)-\left[s(\theta)-s^{*}(\theta)\right]\right\} \wedge d\left\{s(\theta)+s^{*}(\theta)-\left[s(\theta)-s^{*}(\theta)\right]\right\} \\
=\frac{1}{4} \int_{\alpha}^{\beta}\left\{\left[s(\theta)+s^{*}(\theta)\right] \wedge d\left[s(\theta)-s^{*}(\theta)\right]+\left[s(\theta)-s^{*}(\theta)\right] \wedge d\left[s(\theta)+s^{*}(\theta)\right]\right\} .
\end{gathered}
$$

The same argument as in (i) shows that

$$
\int_{\alpha}^{\beta}\left[s(\theta)-s^{*}(\theta)\right] \wedge d\left[s(\theta)+s^{*}(\theta)\right]=0,
$$

and hence that

$$
0=\int_{\alpha}^{\beta} d\left\{\left[\left(s(\theta)+s^{*}(\theta)\right] \wedge\left[s(\theta)-s^{*}(\theta)\right]\right\}=2 s(\alpha) \wedge s^{*}(\alpha)-2 s(\beta) \wedge s^{*}(\beta) .\right.
$$

Lemma 2.8. Suppose that $X$ fulfills (1) and that $s:[0,2 \pi] \rightarrow S$ is a natural map for $S$. Then:

(i) $s$ is continuously differentiable, and there is a continuous function $p:[0,2 \pi] \rightarrow$ $\mathbb{R}_{+}$such that $s^{\prime}(\theta)=p(\theta) s^{\perp}(\theta)$.

(ii) For every $\theta \in[0,2 \pi], s^{\prime}(\theta) \wedge s^{*}(\theta)>0$.

(iii) $s^{*}$ is continuously differentiable, and there is a continuous function $q$ : $[0,2 \pi] \rightarrow \mathbb{R}_{+}$such that $s^{* \prime}(\theta)=q(\theta) s^{* \perp}(\theta)$.

Proof. (i). Since $X$ fulfills (1) it is smooth, and it is well known that this is equivalent to the continuous differentiability of $x \in X \rightarrow\|x\|$ at $X \backslash\{0\}$. Then it follows from

$$
s(\theta)=\|(\cos \theta, \sin \theta)\|^{-1}(\cos \theta, \sin \theta)
$$

that $s$ is continuously differentiable.

Also, the fact that

$$
s(\theta) \wedge s^{\perp}(\theta)=\sup \left\{s(\lambda) \wedge s^{\perp}(\theta): \lambda \in[0,2 \pi]\right\}
$$


shows that $s^{\prime}(\theta) \wedge s^{\perp}(\theta)=0$. Furthermore, it follows from

$$
\begin{aligned}
& \|(\cos \theta, \sin \theta)\|^{2} s_{1}^{\prime}(\theta)=-\sin \theta\|(\cos \theta, \sin \theta)\|-\cos \theta\|(\cos \theta, \sin \theta)\|^{\prime}, \\
& \|(\cos \theta, \sin \theta)\|^{2} s_{2}^{\prime}(\theta)=\cos \theta\|(\cos \theta, \sin \theta)\|-\sin \theta\|(\cos \theta, \sin \theta)\|^{\prime},
\end{aligned}
$$

that $s^{\prime}(\theta) \neq 0$, and it is obvious that $s(\theta) \prec s^{\prime}(\theta)$. I.e., $s^{\prime}(\theta)=p(\theta) s^{\perp}(\theta)$, with $p(\theta)>0$.

Finally, the continuity of $p$ follows from the continuity of $s^{\prime}$ and $s^{\perp}$.

(ii). We saw in Lemma 2.7 that, for any $0 \leq \alpha<\beta \leq 2 \pi$,

$$
\int_{\alpha}^{\beta}\left[s^{*}(\theta)-s^{* *}(\theta)\right] \wedge d\left[s^{*}(\theta)+s^{* *}(\theta)\right]=-\int_{\alpha}^{\beta}\left[2 s^{*}(\theta)+s(\theta)\right] \wedge d s(\theta)=0,
$$

i.e.,

$$
2 \int_{\alpha}^{\beta} s^{*}(\theta) \wedge d s(\theta)=-\int_{\alpha}^{\beta} s(\theta) \wedge d s(\theta)<0 .
$$

Then, since $s$ is continuously differentiable,

$$
\int_{\alpha}^{\beta} s^{*}(\theta) \wedge s^{\prime}(\theta) d \theta<0
$$

as we wished to show.

(iii). It follows from Lemma 2.7(iii) that, for any $\lambda \neq \theta$,

$$
0=\frac{s(\lambda)-s(\theta)}{\lambda-\theta} \wedge s^{*}(\lambda)+s(\theta) \wedge \frac{s^{*}(\lambda)-s^{*}(\theta)}{\lambda-\theta} .
$$

Hence,

$$
\lim _{\lambda \rightarrow \theta} s(\theta) \wedge \frac{s^{*}(\lambda)-s^{*}(\theta)}{\lambda-\theta}=-s^{\prime}(\theta) \wedge s^{*}(\theta)<0 .
$$

Since $X$ is smooth and $s^{*}$ is continuous, if $\left(\lambda_{n}\right)_{n \in \mathbb{N}}$ and $\left(\bar{\lambda}_{n}\right)_{n \in \mathbb{N}}$ are convergent to $\theta$ sequences such that the sequences

$$
\left(\frac{s^{*}\left(\lambda_{n}\right)-s^{*}(\theta)}{\lambda_{n}-\theta}\right)_{n \in \mathbb{N}}, \quad\left(\frac{s^{*}\left(\bar{\lambda}_{n}\right)-s^{*}(\theta)}{\bar{\lambda}_{n}-\theta}\right)_{n \in \mathbb{N}},
$$

are also convergent, then there exist two positive numbers $q(\theta)$ and $\bar{q}(\theta)$ such that

$$
\lim _{n \rightarrow \infty} \frac{s^{*}\left(\lambda_{n}\right)-s^{*}(\theta)}{\lambda_{n}-\theta}=q(\theta) s^{* \perp}(\theta), \quad \lim _{n \rightarrow \infty} \frac{s^{*}\left(\bar{\lambda}_{n}\right)-s^{*}(\theta)}{\bar{\lambda}_{n}-\theta}=\bar{q}(\theta) s^{* \perp}(\theta),
$$

and it follows from the above result that $q(\theta)=\bar{q}(\theta)$, i.e., that

$$
\lim _{\lambda \rightarrow \theta} \frac{s^{*}(\lambda)-s^{*}(\theta)}{\lambda-\theta}=q(\theta) s^{* \perp}(\theta)=-s^{\prime}(\theta) \wedge s^{*}(\theta) .
$$

Finally, the continuity of $s^{* \prime}$ and $q$ follows from the continuity of $s^{\prime}, s^{*}$, and $s^{* \perp}$.

\section{MAIN RESUlts}

Theorem 3.1. $X$ is an inner product space if and only if

$$
u, v \in S, \inf _{t \in[0,1]}\|t u+(1-t) v\|=\frac{1}{2} \Rightarrow u+v \in S .
$$


Proof. It is easy to see (and well known) that if $X$ is an i.p.s., i.e. $\|x\|^{2}=(x \mid x)$, then it fulfills (1).

It suffices to consider that, for any $u, v \in S$ the convex function

$$
F(t)=\|(1-t) u+t v\|^{2}=1-2 t+2 t^{2}+2 t(1-t)(u \mid v)
$$

is such that

$$
F^{\prime}(t)=2(1-2 t)[(u \mid v)-1]
$$

and hence, when $(u \mid v)<1$ (i.e. when $u$ and $v$ are linearly independent), $F$ attains its minimum at $t=\frac{1}{2}$.

By virtue of the above lemmas, to prove the converse we can take $X$ to be the space $\mathbb{R}^{2}$ endowed with a norm, and we can denote by $u$ and $u^{*}$ the two points $u$ and $v$ of hypothesis (1).

Let $s:[0,2 \pi] \rightarrow S$ be a natural map for $S$. It follows from Lemma 2.7(ii) that, for any $\alpha \in[0,2 \pi]$,

$$
\int_{0}^{\alpha} s(\theta) \wedge d s(\theta)=\int_{0}^{\alpha} s^{*}(\theta) \wedge d s^{*}(\theta),
$$

from Lemma 2.5(ii) and (iv) that

$$
s(\theta) \perp s(\theta)+2 s^{*}(\theta), \quad s^{*}(\theta) \perp-2 s(\theta)-s^{*}(\theta),
$$

and from Lemma 2.8 that $s$ and $s^{*}$ are continuously differentiable and such that

$$
s^{\prime}(\theta)=p(\theta) s^{\perp}(\theta), \quad s^{* \prime}(\theta)=q(\theta) s^{* \perp}(\theta),
$$

where $p$ and $q$ are positive and continuous functions.

Then it follows from the uniqueness of $s^{\perp}(\theta)$ that there exist two continuous functions $k:[0,2 \pi] \rightarrow \mathbb{R}_{+}$and $l:[0,2 \pi] \rightarrow \mathbb{R}_{+}$such that

$$
s^{\prime}(\theta)=k(\theta)\left[s(\theta)+2 s^{*}(\theta)\right], \quad s^{* \prime}(\theta)=l(\theta)\left[-2 s(\theta)-s^{*}(\theta)\right],
$$

and the first equality between the Riemann-Stieltjes integrals can be reduced to the following equality between ordinary Riemann integrals:

$$
\int_{0}^{\alpha} k(\theta) s(\theta) \wedge\left[s(\theta)+2 s^{*}(\theta)\right] d \theta=\int_{0}^{\alpha} l(\theta) s^{*}(\theta) \wedge\left[-2 s(\theta)-s^{*}(\theta)\right] d \theta,
$$

i.e. (see Lemma $2.7($ iii)),

$$
\int_{0}^{\alpha} k(\theta) d \theta=\int_{0}^{\alpha} l(\theta) d \theta \quad(\alpha \in[0,2 \pi])
$$

from which it follows ( $k$ and $l$ are continuous) that $k=l$.

Hence, we have the following system of differential equations:

$$
\begin{gathered}
s_{1}^{\prime}(\theta)=k(\theta)\left[s_{1}(\theta)+2 s_{1}^{*}(\theta)\right], \\
s_{2}^{\prime}(\theta)=k(\theta)\left[s_{2}(\theta)+2 s_{2}^{*}(\theta)\right], \\
s_{1}^{* \prime}(\theta)=-k(\theta)\left[2 s_{1}(\theta)+s_{1}^{*}(\theta)\right], \\
s_{2}^{* \prime}(\theta)=-k(\theta)\left[2 s_{2}(\theta)+s_{2}^{*}(\theta)\right] .
\end{gathered}
$$

The first and third give that

$$
2 s_{1}(\theta) s_{1}^{\prime}(\theta)+s_{1}^{*} s_{1}^{\prime}(\theta)+s_{1}(\theta) s_{1}^{* \prime}(\theta)+2 s_{1}^{*}(\theta) s_{1}^{* \prime}(\theta)=0,
$$

i.e. that $s_{1}^{2}(\theta)+s_{1}^{* 2}(\theta)+s_{1}(\theta) s_{1}^{*}(\theta)$ is constant.

Analogously, the second and fourth give that $s_{2}^{2}(\theta)+s_{2}^{* 2}(\theta)+s_{2}(\theta) s_{2}^{*}(\theta)$ is also constant. 
Then, for the (non-restrictive) initial data

$$
\left(s_{1}(0), s_{2}(0)\right)=(1,0), \quad\left(s_{1}^{*}(0), s_{2}^{*}(0)\right)=\left(-\frac{1}{2}, \frac{\sqrt{3}}{2}\right),
$$

we have that

$$
s_{1}^{2}(\theta)+s_{1}^{* 2}(\theta)+s_{1}(\theta) s_{1}^{*}(\theta)=s_{2}^{2}(\theta)+s_{2}^{* 2}(\theta)+s_{2}(\theta) s_{2}^{*}(\theta)=\frac{3}{4} .
$$

This, together with

$$
s_{1}(\theta) s_{2}^{*}(\theta)-s_{2}(\theta) s_{1}^{*}(\theta)=\frac{\sqrt{3}}{2}
$$

(see Lemma 2.7 (iii)), leads to

$$
s_{1}^{2}(\theta)+s_{2}^{2}(\theta)=1
$$

i.e., to the fact that $S$ is a circumference (an ellipse), as we wished to show.

Theorem 3.2. Suppose that $X$ is smooth and of dimension $\geq 3$. Then $X$ is an inner product space if and only if

$$
u, v, w \in S, u+v+w=0 \Rightarrow 0 \in Z_{X}(u, v, w) .
$$

Proof. It is known (see, e.g., 4], p. 238) that if $X$ is an inner product space (of any dimension), i.e. $\|x\|^{2}=(x \mid x)$, then it fulfills (2). Indeed, let $u, v, w \in S$ be such that $u+v+w=0$. Then for

$$
\begin{aligned}
F(x) & =\|u-x\|+\|v-x\|+\|w-x\| \\
& =\sqrt{(u-x \mid u-x)}+\sqrt{(v-x \mid v-x)}+\sqrt{(u+v+x \mid u+v+x)},
\end{aligned}
$$

we have that

$$
F^{\prime}(x)(t)=\frac{(x-u \mid t)}{\sqrt{(u-x \mid u-x)}}+\frac{(x-v \mid t)}{\sqrt{(v-x \mid v-x)}}+\frac{(x+u+v \mid t)}{\sqrt{(u+v+x \mid u+v+x)}},
$$

from which it follows that

$$
F^{\prime}(0)(t)=-(u \mid t)-(u \mid t)+(u+v \mid t)=0,
$$

i.e., the convex function $F$ attains its minimum at 0 .

To prove the converse we may assume $\operatorname{dim} X=3$.

Since $X$ is smooth, for any $u \in S$ there is a unique $u^{\prime} \in S^{\prime}$ (unit sphere of the dual space $\left.X^{\prime}\right)$ such that $u^{\prime}(u)=1$.

Let $u, v, w \in S$ be such that $u+v+w=0$. Then $0 \in Z_{X}(u, v, w)$, and a corollary of the Hahn-Banach theorem (see, e.g., 2, Proposition 1) says that this is equivalent to $u^{\prime}+v^{\prime}+w^{\prime}=0$.

So we have that

$$
\begin{gathered}
u^{\prime}(u)=v^{\prime}(v)=w^{\prime}(w)=1, \\
u^{\prime}(u+v+w)=v^{\prime}(u+v+w)=w^{\prime}(u+v+w)=0, \\
\left(u^{\prime}+v^{\prime}+w^{\prime}\right)(u)=\left(u^{\prime}+v^{\prime}+w^{\prime}\right)(v)=\left(u^{\prime}+v^{\prime}+w^{\prime}\right)(w)=0,
\end{gathered}
$$

from which it follows that

$$
\begin{aligned}
u^{\prime}(v)+u^{\prime}(w) & =v^{\prime}(u)+v^{\prime}(w)=w^{\prime}(u)+w^{\prime}(v)=-1, \\
v^{\prime}(u)+w^{\prime}(u) & =u^{\prime}(v)+w^{\prime}(v)=u^{\prime}(w)+v^{\prime}(w)=-1,
\end{aligned}
$$

and, hence,

$$
u^{\prime}(v)=v^{\prime}(w)=w^{\prime}(u), \quad u^{\prime}(w)=v^{\prime}(u)=w^{\prime}(v) .
$$


Let $L$ be the 2-dimensional subspace $\operatorname{span}(u, v, w)$, let $\left(L_{n}\right)$ be a sequence of 2-dimensional subspaces of $X$ that contain $u$ and converges (in the obvious sense) to $L$, and let $v_{n}, w_{n} \in L_{n} \cap S$ be such that

$$
u+v_{n}+w_{n}=0 .
$$

Since the sequence

$$
\left(\tau_{n},-\tau_{n}\right)=\left(\frac{v-v_{n}}{\left\|v-v_{n}\right\|}, \frac{w-w_{n}}{\left\|w-w_{n}\right\|}\right)=\left(\frac{v-v_{n}}{\left\|v-v_{n}\right\|}, \frac{v_{n}-v}{\left\|v_{n}-v\right\|}\right)
$$

is in the compact set $S \times S$ it has a subsequence that converges to a point $(\tau,-\tau) \in$ $S \times S$ such that $v \perp \tau$ and $w \perp \tau$, i.e. (see Remark 2.2) $v^{\prime}(\tau)=w^{\prime}(\tau)=0$ and thus $u^{\prime}(\tau)=0$.

Moreover, since $\operatorname{dim}\left(\operatorname{ker} u^{\prime} \cap \operatorname{ker} v^{\prime} \cap \operatorname{ker} w^{\prime}\right)=1$, every convergent subsequence of $\left(\tau_{n},-\tau_{n}\right)$ converges to either $(\tau,-\tau)$ or $(-\tau, \tau)$, and hence

$$
\lim _{n \rightarrow \infty} u^{\prime}\left(\frac{v-v_{n}}{\left\|v-v_{n}\right\|}\right)=\lim _{u+\bar{v} \in S, \bar{v} \rightarrow v} u^{\prime}\left(\frac{v-\bar{v}}{\|v-\bar{v}\|}\right)=0 .
$$

Since this is valid for every $v \in S$ such that $u+v \in S$, we get that $\{v \in S$ : $u+v \in S\}$ is a differentiable curve that is contained in a plane parallel to $\operatorname{ker} u^{\prime}(\tau$ is a tangent vector at $v$ and $\left.u^{\prime}(\tau)=0\right)$. Specifically,

$$
\{v \in S: u+v \in S\}=\left(-\frac{1}{2} u+\operatorname{ker} u^{\prime}\right) \cap S,
$$

i.e. $u^{\prime}(v)=u^{\prime}(w)=-1 / 2$, for every $u, v, w \in S$ such that $u+v+w=0$.

We have, finally, that for every $\lambda \in \mathbb{R}$

$$
\|v+w\|=-u^{\prime}(v+w+\lambda(v-w)) \leq\|v+w+\lambda(v-w)\|,
$$

i.e., $v+w \perp v-w$.

In other words, $w=v^{*}$ and the segment $[v, w]$ supports $\frac{1}{2} S$ at its middle point. Since this is true for every $v \in S$, Theorem 3.1 shows that $X$ is an inner product space.

Remark 3.3. We presume that in the above theorem the smoothness of $X$ is unnecessary, but the corresponding proof appears to be much more involved.

Remark 3.4. In a first version of this paper we said that $u+v+w=0$ and $u^{\prime}+v^{\prime}+w^{\prime}=0$ imply $u^{\prime}(v)=u^{\prime}(w)=-1 / 2$, and then we concluded that if $X$ (of any dimension) fulfils

$$
u, v, w \in S, u+v+w=0 \Rightarrow 0 \in Z_{\mathrm{co}(u, v, w)}(u, v, w),
$$

then $X$ is an inner product space.

But for $\operatorname{dim} X=2$ we only have $u^{\prime}(v)+u^{\prime}(w)=-1$, and, furthermore, the following counterexample shows that not only our old proof was wrong.

Example $3.5([5])$. Let $X$ be the space $\mathbb{R}^{2}$ endowed with a norm whose unit sphere $S$ is a (rectilinear or curvilinear) regular hexagon, i.e. a convex curve that is invariant under rotations of $\pi / 3$. Then, it is easy to see that if $u, v, w \in S$ are such that $u+v+w=0$, then they are vertices of an equilateral triangle inscribed in $S$.

Furthermore, if, for the above three points, $z \in Z_{X}(u, v, w)$, then either $z=0$ or $z$ is a vertex of an equilateral triangle, centered at 0 , whose other vertices are also in $Z_{X}(u, v, w)$, and, since this set is convex, $0 \in Z_{X}(u, v, w)$. 
Note finally that if $S$ is a rectilinear regular hexagon, then $X$ is neither smooth nor rotund, but for other curvilinear regular hexagons $X$ may be smooth and rotund.

\section{ACKNOWLEDGEMENT}

The authors thank Professor Chelidze for the above counterexample, and Professor Papini and the referee of the first version of this paper for their good idea of

considering spaces of dimension $\geq 3$ in the last theorem. We also thank the referee of the second version for his/her kind and accurate hints.

\section{REFERENCES}

1. D. AmIR, "Characterization of Inner Product Spaces", Birkhauser Verlag, Basel, 1986. MR0897527 (88m:46001)

2. C. Benítez, M. Fernández, and M.L. Soriano, Location of the Fermat-Torricelli medians of three points, Trans. Amer. Math. Soc. 354 (2002), 5027-5038. MR1926847(2003h:46032)

3. G. Birkhoff, Orthogonality in linear metric spaces, Duke Math. J. 1 (1935), 169-172. MR1545873

4. V. Boltyanski, H. Martini, and V. Soltan, "Geometric Methods and Optimization Problems", Kluwer Academic Publishers, Dordrecht, 1999. MR.1677397 (2000c:90002)

5. G. Chelidze, personal communication.

6. D. Cieslik, "Steiner Minimal Trees", Kluwer Academic Publishers, Dordrecht, 1998. MR.1617288(99i:05062)

7. M.M. DAY, Some characterizations of inner-product spaces. Trans. Amer. Math. Soc. 62 (1947), 320-337. MR0022312 (9:192c)

8. N.I. GuRARi AND Y.I. Sozonov, On normed spaces which have no bias of the unit sphere, Math. Notes 7 (1970), 187-189. MR0264375 (41:8971)

9. R.C. James, Orthogonality and linear functionals in normed linear spaces, Trans. Amer. Math. Soc. 61 (1947), 265-292. MR0021241(9:42c)

Departamento de Matemáticas, Universidad de Extremadura, 06071 Badajoz, Spain

E-mail address: cabero@unex.es

Departamento de Matemáticas, Universidad de Extremadura, 06071 Badajoz, Spain

E-mail address: dyanez@unex.es 